\title{
Hypoxia-inducible factor-1 mediates the biological effects of oxygen on human trophoblast differentiation through TGF $\beta_{3}$
}

\author{
Isabella Caniggia, ${ }^{1,2,3}$ Homa Mostachfi, ${ }^{2}$ Jennifer Winter, ${ }^{2,3}$ Max Gassmann, ${ }^{4}$ \\ Stephen J. Lye, ${ }^{2,3}$ Maciej Kuliszewski, ${ }^{1}$ and Martin Post ${ }^{1,3}$ \\ ${ }^{1}$ Department of Pediatrics, The Hospital for Sick Children Research Institute, Hospital for Sick Children, \\ University of Toronto, Toronto, Ontario, Canada M5G 1X8 \\ ${ }^{2}$ Program in Development and Fetal Health, Samuel Lunenfeld Research Institute, Department of Obstetrics and \\ Gynaecology, Mount Sinai Hospital, Toronto, Ontario, Canada M5G 1X5 \\ ${ }^{3}$ Department of Physiology, University of Toronto, Toronto, Ontario, Canada \\ ${ }^{4}$ Institute of Physiology, University of Zurich-Irchel, CH-8057 Zurich, Switzerland \\ Address correspondence to: Isabella Caniggia, Mount Sinai Hospital, Samuel Lunenfeld Research Institute, \\ 600 University Avenue, Toronto, Ontario, Canada M5G 1X5. \\ Phone: (416) 586-4803; Fax: (416) 586-8745; E-mail: Caniggia@mshri.on.ca.
}

Received for publication September 1, 1999, and accepted in revised form January 21, 2000.

\begin{abstract}
During early pregnancy, placentation occurs in a relatively hypoxic environment that is essential for appropriate embryonic development. Intervillous blood flow increases around 10 to 12 weeks of gestation and results in exposure of trophoblast cells to increased oxygen tension. Before this time, low oxygen appears to prevent trophoblast differentiation toward an invasive phenotype. Using human villous explants of 5-8 weeks' gestation, we found that low oxygen tension triggered trophoblast proliferation, fibronectin synthesis, $\alpha_{5}$ integrin expression, and gelatinase A activity. These biochemical markers were barely detectable under oxic conditions. We therefore examined the placental expression of hypoxia-inducible factor-1 (HIF-1), a master regulator of oxygen homeostasis, and determined that expression of HIF- $1 \alpha$ subunit during the first trimester of gestation parallels that of $\mathrm{TGF}_{3}$, an inhibitor of extravillous trophoblast differentiation. Expression of both molecules is high in early pregnancy and falls around 9 weeks of gestation, when placental $\mathrm{pO}_{2}$ levels are believed to increase. Increasing oxygen tension induced a similar decrease in expression in cultured explants. Moreover, antisense inhibition of HIF- $1 \alpha$ expression in hypoxic explants inhibited expression of TGF $\beta_{3}$, arrested cell proliferation, decreased $\alpha_{5}$ expression and gelatinase $\mathrm{A}$ activity, and triggered biochemical markers of an invasive trophoblast phenotype such as $\alpha_{1}$ integrin and gelatinase $B$ expression. These data suggest that the oxygen-regulated early events of trophoblast differentiation are in part mediated by TGF $\beta_{3}$ through HIF-1 transcription factors.
\end{abstract}

J. Clin. Invest. 105:577-587 (2000).

\section{Introduction}

During placentation cytotrophoblast cells localized in floating and anchoring villi follow 2 distinct pathways of differentiation $(1,2)$. Villous cytotrophoblasts fuse to form the highly specialized syncytiotrophoblast layer that contributes to gas, nutrient, and waste exchange. In anchoring villi cytotrohoblast generates a multilayered column of highly invasive extravillous trophoblasts [EVT] that later migrate into the decidua and invade the first third of the myometrium. Within the myometrium the EVT induce remodeling of the spiral arterioles to produce the low-resistance vascular system that is essential for fetal growth (3). This period in development is characterized by an important physiological switch in oxygen tension at the opening of the intervillous space.

During the first weeks of gestation EVT exists in a relatively low-oxygen environment. Maternal blood flow to the placenta is limited and endovascular EVT inva- sion is minimal (4). This low-oxygen environment is essential for normal embryonic and placental development because the early conceptus has little protection against oxygen-generated free radicals. Genbacev et al. (5) have provided in vitro evidence to support a role for low oxygen tension in maintaining trophoblasts in a proliferative, noninvasive, and immature phenotype. In mammalian systems, the adaptive response to hypoxia is accompanied by an increase in the expression of a variety of genes, including the hematopoietic growth factor erythropoietin gene, vascular endothelial growth factor, glycolytic enzymes, and inducible nitric oxide synthetase (6-9). Most of these genes are regulated by a common oxygen-sensing pathway, the formation of the hypoxia-inducible factor-1 (HIF-1) protein complex $(10,11)$. HIF-1, a basic helix-loop-helix PAS (bHLHPAS) transcription factor, binds to a short DNA motif identified in the 5 -flanking regions of many of the hypoxia-induced genes (12). HIF-1 binds DNA as a het- 
eromeric complex composed of 2 subunits, the constitutively expressed HIF-1 $\beta$ (ARNT) and HIF- $1 \alpha$, which is present in hypoxic conditions and is rapidly degraded by the proteasome under normoxic conditions through an interaction with the tumour suppressor protein (von Hippel-Lindau) VHL (13). There are no data on the expression of HIF- $1 \alpha$ in the placenta or on its role in regulating trophoblast differentiation.

Around 10 to 12 weeks of gestation there is a critical physiologic increase in oxygen tension as the intervillous space opens and the conceptus is exposed to maternal blood. It is at this time that EVT differentiates towards a more invasive phenotype. We demonstrated recently that $\mathrm{TGF} \beta_{3}$ is highly expressed during early placentation (6-8 weeks) when oxygen tension is low and declines at the end of the first trimester (10-12 weeks) when oxygen tension increases (14). TGF $\beta_{3}$ inhibits the early events of trophoblast differentiation along the invasive pathway. In pregnancies complicated by earlyonset preeclampsia, $\mathrm{TGF}_{3}$ expression remains abnormally elevated and trophoblasts are arrested to an intermediate immature phenotype. The mechanisms that regulate expression of placental TGF $\beta_{3}$ during the first trimester of gestation remain to be determined.

Based on these data we hypothesize that early in the first trimester ( $<10$ weeks) the low oxygen tension environment maintains trophoblasts in a relatively immature, proliferative state, mediated by $\mathrm{TGF}_{3}$ through HIF-1 $\alpha$. Subsequently, trophoblast exposure to increased oxygen tension reduces HIF- $1 \alpha$ and TGF $\beta_{3}$ expression, which in turn releases the block to EVT differentiation and invasion into the uterine wall. Data presented herein provide support for this hypothesis.

\section{Methods}

Human chorionic villous explant culture. Villous explant cultures were established from first-trimester human placentas obtained from elective terminations of pregnancies as described previously (15). Placental tissue from 5 to 14 weeks of gestation was dated according to the criteria of the Carnegie classification evaluating the length of the embryo and external characteristics of embryonic/fetal parts. Briefly, placental tissue was placed in icecold PBS and processed within 2 hours of collection. The tissue was aseptically dissected to remove decidual tissue and fetal membranes. Small fragments of placental villi (15-25 mg wet weight) were teased apart and placed on Millicell-CM culture dish inserts (Millipore Corp, Bedford, Massachusetts, USA), precoated with $0.2 \mathrm{~mL}$ of undiluted Matrigel (Collaborative Biomedical Products, Bedford, Massachusetts, USA), and placed in a 24-well culture dish. Explants were cultured in serum-free DMEM/F12 (GIBCO-BRL, Grand Island, New York, USA), supplemented with $100 \mu \mathrm{g} / \mathrm{mL}$ streptomycin, 100 $\mathrm{U} / \mathrm{mL}$ penicillin, and $0.25 \mu \mathrm{g} / \mathrm{mL}$ ascorbic acid, $\mathrm{pH}$ 7.4. Villous explants were placed at $37^{\circ} \mathrm{C}$ in either standard tissue culture condition $\left(5 \% \mathrm{CO}_{2}\right.$ in $95 \%$ air $)$ or maintained in an atmosphere of $3 \% \mathrm{O}_{2} / 93 \% \mathrm{~N}_{2} / 5 \% \mathrm{CO}_{2}$. Morphological integrity and viability of villous explants and their EVT differentiation were monitored daily for up to 6 days as reported previously (15).

Antisense oligonucleotides and their effects on trophoblast differentiation along the invasive pathway. Phosphorothioate oligonucleotides (ON) were synthesized on a DNA synthesizer and purified by capillary electrophoresis. Oligonucleotides of $15 \mathrm{bp}$ targeted against sequences adjacent to the AUG initiation codon of human TGF $\beta_{3}$ and HIF- $1 \alpha$ mRNA were synthesized. Previous studies have demonstrated that antisense oligonucleotides targeted to sequences adjacent to initiation codons are most efficient in inhibiting translation (16). Furthermore, 16-mer oligonucleotides are short enough to be taken up efficiently and provide sufficient specificity for hybridization to the corresponding target mRNA (16). The sequences of the antisense and sense HIF- $1 \alpha$ and TGF $\beta_{3}$ oligonucleotides were HIF- $1 \alpha$ : $5^{\prime}$-GCCGGCGCCCTCCAT-3' and 5'-ATGGAGGGCGCCGGC-3'; TGF $\beta_{3}$ : 5'CCTTTGCAAGTGCATC- $3^{\prime}$ and $5^{\prime}$-GATGCACTTGCAAAGG$3^{\prime}$, respectively. Oligonucleotides were dissolved in water and their concentration was estimated by optical density at $\mathrm{OD}_{260}$. Villous explants, prepared from placentas of 5-14 weeks' gestation were incubated in DMEM/F12 at $3 \% \mathrm{O}_{2}$ overnight and then incubated in medium alone or medium containing antisense or sense oligonucleotides $(10 \mu \mathrm{M})$. Culture media with and without oligonucleotides were routinely changed every 48 hours.

RT-PCR and Southern blot analysis. Total RNA was extracted from placental tissue and from villous explants, reverse transcribed, and amplified by 15 cycles of PCR using HIF- $1 \alpha$ - and TGF $\beta_{3}$-specific primers. RTPCR products were analysed by Southern blotting using TGF $\beta 3$ and HIF- $1 \alpha$ cDNAs labeled with ${ }^{32} \mathrm{P}(17)$. The primer sets chosen for amplification of TGF $\beta 3$ and HIF-1 $\alpha$ were based on human mRNA sequences. Primers used for amplification were: (a) $T G F \beta_{3}$ cDNA: (forward primer) 5'-CAAAGGGCTCTGGTGGTCCTG-3', (reversed primer) 5'-CTTGGAGGTAATTCCTTTAGGG-3' (predicted product size $=374 \mathrm{bp}$ ); (b) HIF-1 $\alpha \mathrm{cDNA}$ : (forward primer) 5'- ATCTCGGCGAAGTAAAGAATCTG-3', (reversed primer) 5'-GTCACCATCATCT GTGAGAACC-3' (predicted product size $=243 \mathrm{bp}$ ); (c) $\beta$-actin cDNA: (forward primer) 5'- CTTCTACAATGAGCTGGGTG-3', (reversed primer) 5'-TCATGAGGTAGTCAGTCAGG-3' (predicted product size $=307 \mathrm{bp}$ ). The identity of the PCR reaction products was confirmed by sequencing. The RT-PCR assay was linear for up to 25 cycles of amplification, and no signals were detected without the initial addition of reverse transcriptase.

Immunohistochemistry. Placental tissue from first trimester (5-14 weeks) and from villous explants, kept in culture for 6 days at either 3 or $20 \% \mathrm{O}_{2}$, was fixed for $2-4$ hours at $4^{\circ} \mathrm{C}$ in $4 \%(\mathrm{vol} / \mathrm{vol})$ paraformaldehyde, embedded in paraffin, and cut into 5-micron sections. To verify the quality of the tissue and select the most representative sections, every 10 th one was stained with hematoxylin and eosin. Neighbouring sections were selected and stained using the avidin-biotin immunoperoxidase method. Endogenous peroxidase enzyme 
activity was quenched with $1.5 \%$ (vol/vol) hydrogen peroxide in methanol for 30 minutes. Antigen was retrieved by incubation with $0.125 \%$ trypsin in PBS for $10 \mathrm{~min}$ utes at room temperature. Nonspecific binding sites were blocked using $5 \%$ (vol/vol) normal goat serum (NGS) and 1\% (wt/vol) BSA in Tris buffer for $30 \mathrm{~min}-$ utes at room temperature. Optimal antibody concentrations were established in preliminary experiments by titration. A purified mouse $\mathrm{mAb}$ directed against human Ki67 (Boehringer Mannheim, Montreal, Quebec, Canada) and rat mAb to cytokeratin (7D3; gift of Jay Cross, Samuel Lunenfeld Research Institute) were used, each at 1:20 dilution, whereas the mouse $\mathrm{mAb}$ to human MMP9 (mAb Ab-2; Oncogene Research Products, Calbiochem, Cambridge, Massachusetts, USA) and $\mathrm{mAb}$ to human $\alpha 1$ integrin (VLA-1; T Cell Diagnostics,
Woburn, Massachusetts, USA) were used at 1:100 and 1:10 dilution, respectively. Mouse mAbs against HIF- $1 \alpha$ $(\mathrm{mgc} 3)(18)$ were used at 1:50 dilution and mouse mAbs against $\alpha_{5}$ integrin subunit (P1D6; Chemicon International, Temecula, California, USA) were used at 1:200 dilution. For the HIF-1 $\alpha$ staining, antigen was retrieved by microwaving the section in $10 \mathrm{mM}$ sodium citrate, $\mathrm{pH}$ 6.0, for 5 minutes, and endogenous peroxidase was quenched using 3\% (vol/vol) hydrogen peroxide in methanol for 30 minutes. In all the experiments slides were washed 3 times with Tris buffer, $\mathrm{pH}$ 7.6, and incubated with a 30 -fold dilution of biotinylated anti-mouse IgG or a 50-fold dilution of biotinylated anti-rat IgG for 2 hours at room temperature; after washing 3 times with Tris buffer, the slides were incubated with an avidin-biotin complex for 1 hour. Slides were washed a
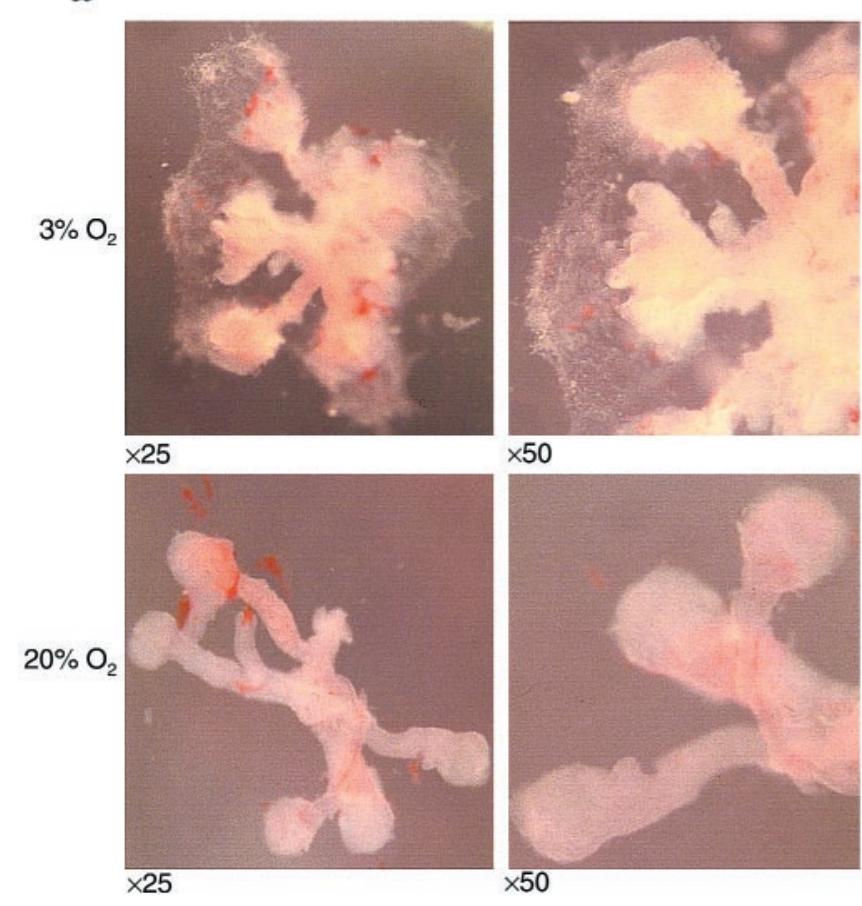

$\times 50$

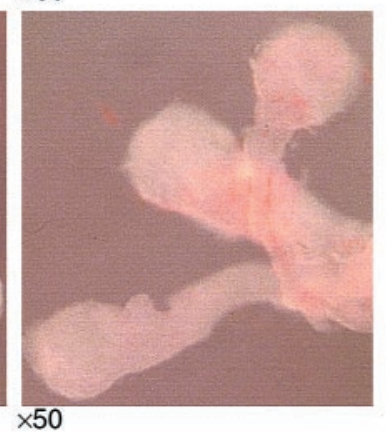

C

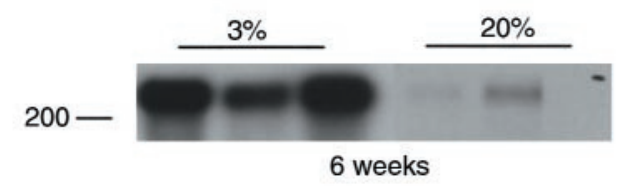

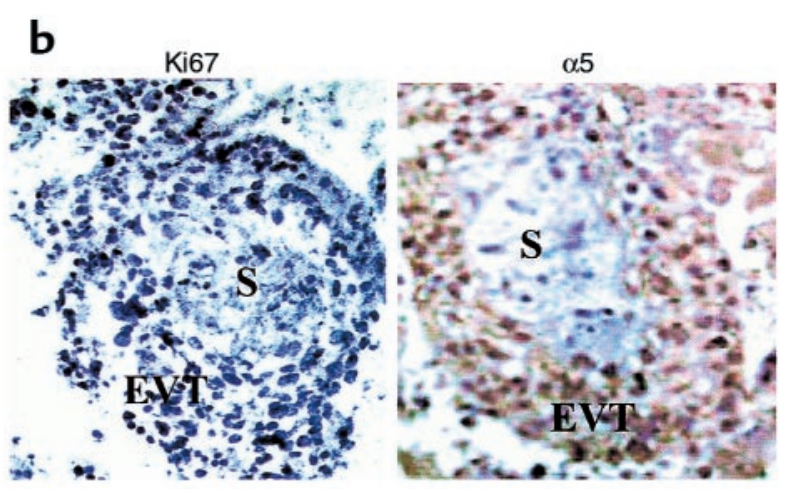
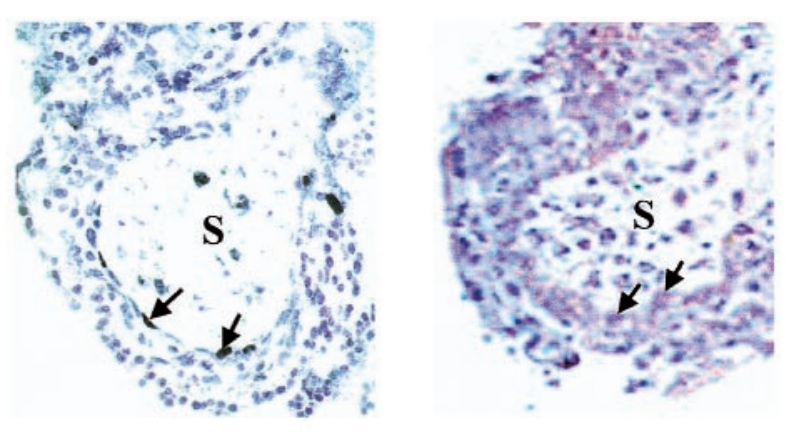

d

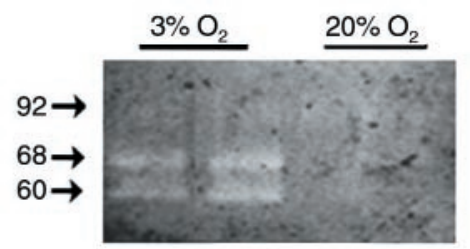

6 weeks

\section{Figure 1}

Low oxygen tension induces trophoblast outgrowth and increases proliferation, fibronectin synthesis, and gelatinase activity in villous explant cultures. (a) Villous explants from 5 to 8 weeks of gestation were maintained in culture for 5 days under normal $\left(20 \% \mathrm{O}_{2}\right)$ or low $\left(3 \% \mathrm{O}_{2}\right)$ oxygen tension. Note that exposure of villous explants to $3 \% \mathrm{O}_{2}$ dramatically increases budding and outgrowth of EVT from the distal end of the villous tips when compared with villous explants kept at $20 \% \mathrm{O}_{2} . \times 25, \times 50$. (b) Section of explants, cultured in either $3 \%$ or $20 \% \mathrm{O}_{2}$, were stained for Ki67, a marker of cellular proliferation and $\alpha 5$ integrin. Strong positive nickel-enhanced immunoreactivity for Ki67 was observed in EVT of the outgrowth (EVT) of explants cultured in $3 \% \mathrm{O}_{2}$. Similar immunolocalization was observed for $\alpha 5$ integrin. A few immunopositive cells for both $\mathrm{Ki} 67$ and $\alpha 5$ integrin were noted in explants kept at $20 \% \mathrm{O}_{2}$ (arrows). S, villous stroma. (c) Explants incubated for 4 days in either low or normal $\mathrm{pO}_{2}$ conditions were metabolically labeled with [ $\left.{ }^{35} \mathrm{~S}\right]$ methionine for 18 hours. Fibronectin was isolated from conditioned medium using gelatin-Sepharose beads. Samples were subjected to SDS-PAGE, and the position of the marker $\left(\right.$ with $\left.M_{r}=200 \times 10^{3}\right)$ is indicated. (d) Samples of medium conditioned by explants, cultured in either 3 or $20 \% \mathrm{O}_{2}$, were collected at day 5 and subjected to analysis by gelatin zymography. Arrows indicate positions of gelatinase activity (gelatinase A/MMP-2: 60 and 68 kDa; gelatinase B/MMP-9: 92 kDa). 


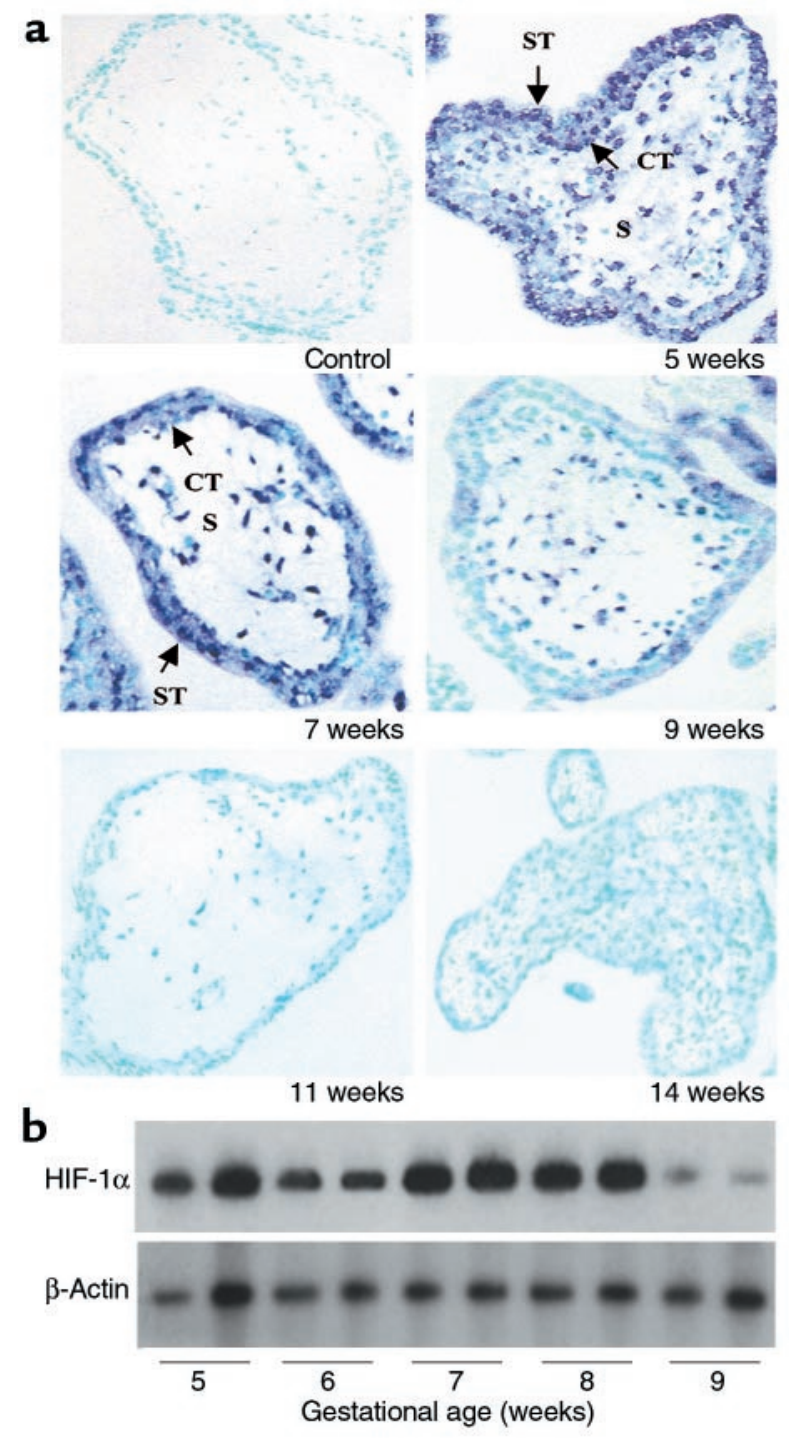

Figure 2

Expression of HIF- $1 \alpha$ in human placenta in the first trimester of gestation (a) Expression of HIF- $1 \alpha$ mRNA was also assessed by in situ hybridization to placental sections at 5-14 weeks of gestation with digoxigenin-labeled sense and antisense HIF- $1 \alpha$ riboprobes. Endogenous alkaline phosphatase was blocked by the addition of levamisole. Sections were counterstained with methyl green. Note that HIF-1 $\alpha$ mRNA expression, revealed by blue staining, is high at 5-7 weeks in chorionic villi, decreases around 9 weeks, and is absent at 11-14 weeks. Controls using a sense HIF- $1 \alpha$ riboprobe were negative. $\times 100$. (b) Message expression of HIF- $1 \alpha$ and TGF $\beta_{3}$ were assessed by low-cycle RTPCR followed by Southern blot analysis using specific probes for HIF$1 \alpha$ and TGF $\beta_{3}$ and the control housekeeping gene $\beta$-actin. Note that mRNA expression of HIF- $1 \alpha$ is high between 5 to 8 weeks of gestation and declines thereafter.

again in Tris buffer and developed in $0.075 \%$ (wt $/ \mathrm{vol}$ ) 3,3-diaminobenzidine in Tris buffer containing $0.002 \%$ (vol/vol) $\mathrm{H}_{2} \mathrm{O}_{2}$, giving rise to a brown product. After light counterstaining with toluidine blue, slides were dehydrated in an ascending ethanol series, cleared in xylene, and mounted. In control experiments, primary antibodies were replaced with blocking solution, $5 \%$ (vol/vol) NGS and 1\% (wt/vol) BSA.
In situ bybridization. Antisense and sense digoxigeninlabeled HIF-1 $\alpha$ riboprobes (17) were generated as described in the RNA-labeling and detection kits (nonradioactive) from Boehringer Mannheim. In situ hybridization to placental tissue from the first trimester (5-14 weeks) was performed according to Braissant and Wahli (19).

Fibronectin synthesis and release. Villous explants of 5-14 weeks' gestation were incubated overnight at $37^{\circ} \mathrm{C}$ in DMEM/F12. Explants were placed in either standard tissue culture condition $\left(5 \% \mathrm{CO}_{2}\right.$ in $95 \%$ air) or maintained in an atmosphere of $3 \% \mathrm{O}_{2} / 93 \% \mathrm{~N}_{2} / 5 \% \mathrm{CO}_{2}$. The medium was changed on various days of culture and replaced by methionine/cysteine-free DMEM containing 25 $\mu \mathrm{Ci} / \mathrm{mL}$ of $\left[{ }^{35} \mathrm{~S}\right]$ methionine/cysteine. The cultures were metabolically labeled for 18 hours. Conditioned culture media were collected and diluted with an equal amount of $25 \mathrm{mM}$ Tris- $\mathrm{HCl}$ buffer, $\mathrm{pH} 7.4,0.15 \mathrm{M} \mathrm{NaCl}$, and $0.5 \%$ (vol/vol) Triton X-100 (Tris/Triton X-100 buffer). Fibronectin was isolated using gelatin-Sepharose as described previously $(15,20)$. Briefly, $50 \mu \mathrm{L}$ of the gelatin-Sepharose suspension was added to $500 \mu \mathrm{L}$ of medi$\mathrm{um}$, and the samples were incubated overnight at $4^{\circ} \mathrm{C}$. The gelatin-Sepharose beads were centrifuged and washed 3 times in Tris/Triton X-100 buffer. Fibronectin was eluted by boiling for 5 minutes in 1\% (vol/vol) SDS and electrophoresed on $4-12 \%$ (wt/vol) polyacrylamide gradient gels. Radiolabeled fibronectin was revealed by autoradiography and quantitated using a PhosphoImager (410A and Image Quant software; Molecular Dynamics, Sunnyvale, California, USA).

Detection of metalloproteases by zymography. Analysis of gelatinolytic activity was performed using $10 \%$ (wt/vol) polyacrylamide gel impregnated with $0.1 \%$ (wt/vol) gelatin (Novex, San Diego, California, USA) as described previously (21). Four microliters of conditioned media harvested from the explant cultures at day 1 and 3 of treatment were mixed with $10 \%$ (vol/vol) glycerol, $2 \%$ (wt/vol) SDS, $0.0025 \%$ (wt/vol) bromophenol blue, $0.5 \mathrm{M}$ Tris, pH 6.8, and subjected to substrate-gel electrophoresis. Gels were then washed twice in $2 \%(\mathrm{vol} / \mathrm{vol})$ Triton X100 for 30 minutes at room temperature to remove the SDS. Then the gels were equilibrated with developing buffer $\left(50 \mathrm{mM}\right.$ Tris-HCl, $0.2 \mathrm{M} \mathrm{NaCl}, 5 \mathrm{M} \mathrm{CaCl}_{2}$, Brij 35, $\mathrm{pH}$ 7.2) for 30 minutes and incubated overnight with the same buffer at $37^{\circ} \mathrm{C}$. Gels were then stained with $0.1 \%$ (wt/vol) Coomassie brilliant blue G-250 after destaining to reveal zones of gelatinase activity.

Western blot analysis. For Western blot analysis of metalloprotease expression, $5 \mu \mathrm{L}$ of conditioned media were subjected to gel electrophoresis using 10\% (wt/vol) polyacrylamide gels (SDS-PAGE). Proteins were then blotted to Western PVDF membrane. Primary antibodies were used at 1:100 dilution (mouse mAb to human MMP9, mAb Ab-2, and MMP2, mAb 75-7F7), detected using horse radish peroxidase-conjugated anti-mouse IgG (1:10.000-fold dilution), and enhanced by chemiluminescence (ECL; Amersham Pharmacia Biotech, Oakville, Ontario, Canada). 
For Western blot analysis of HIF- $1 \alpha, 100 \mu \mathrm{g}$ of 7-15week placental proteins were subjected to $10 \%$ (wt/vol) SDS-PAGE. After electrophoresis, proteins were transferred to nitrocellulose membranes. Nonspecific binding was blocked by incubation in 5\% (wt/vol) nonfat dry milk in Tris-buffered saline containing $0.1 \%$ (vol/vol) Tween-20 (TBST) for 60 minutes. Membranes were then incubated with 1:1000 diluted anti-HIF-1 $\alpha$ $\mathrm{mAb}(\mathrm{mcg} 3)$ in $5 \%(\mathrm{wt} / \mathrm{vol})$ nonfat dry milk in TBST at $4{ }^{\circ} \mathrm{C}$. After overnight incubation, membranes were washed with TBST and incubated for 60 minutes at room temperature with 1:1000 diluted horseradish peroxidase-conjugated anti-mouse IgG in 5\% (wt/vol) nonfat dry milk in TBST. After washing with TBST, blots were detected with chemiluminescent reagent.

Statistical analysis. All data are presented as means \pm SE of at least 3 separate experiments carried out in triplicate. Statistical significance was determined by Student's $t$ test for paired groups and by 1-way ANOVA followed by assessment of differences using Student-Newman-Keuls test for nonpaired groups. Significance was defined as $P<0.05$.

\section{Results}

Low oxygen tension induces the onset of trophoblast proliferation in villous explants. Intervillous blood flow is limited in early placentation but increases at around 10 to 12 weeks of gestation, resulting in exposure of the trophoblast to increased oxygen tension. Because recent studies $(5,22)$ have suggested that a switch in oxygen tension regulates trophoblast proliferation and differentiation along the invasive pathway, we first investigated the effect of different oxygen tensions on the early events of trophoblast differentiation. Human villous explants (5-8 weeks of gestation) cultured on Matrigel under normoxic conditions $\left(20 \% \mathrm{O}_{2}\right)$ in serum-free medium remained viable for approximately 7-10 days and morphologically exhibited minimal EVT budding from the distal end of the villous tips. Exposure of villous explants to low oxygen $\left(3 \% \mathrm{O}_{2}\right)$ resulted in an increase in EVT outgrowth from the distal end of the villous tips when compared with explants cultured under normoxic conditions $\left(20 \% \mathrm{O}_{2}\right)$ (Figure 1a). Whereas low oxygen tension increased EVT outgrowth, no migrating cells were visible after villous explant exposure to low oxygen (Figure 1a). The increase in EVT outgrowth in low $\mathrm{pO}_{2}$ was associated with an increase in cell proliferation, assessed by Ki67 immunoreactivity (Figure 1b), $\alpha 5$ integrin expression (Figure 1b), fibronectin synthesis (Figure 1c), and gelatinase A/MMP2 gelatinolytic activity (Figure 1d).

Ontogeny of HIF- $1 \alpha$ mRNA expression in placenta during the first trimester. To define the mechanism(s) by which low oxygen tension induces an increase in proliferation, fibronectin synthesis, $\alpha 5$ integrin expression, and MMP2 activity, we first studied the expression of HIF$1 \alpha$ in first-trimester placentas using nonradioactive in situ hybridization (Figure 2a) and low-cycle RT-PCR, followed by Southern blot analysis (Figure 2b). Expres-
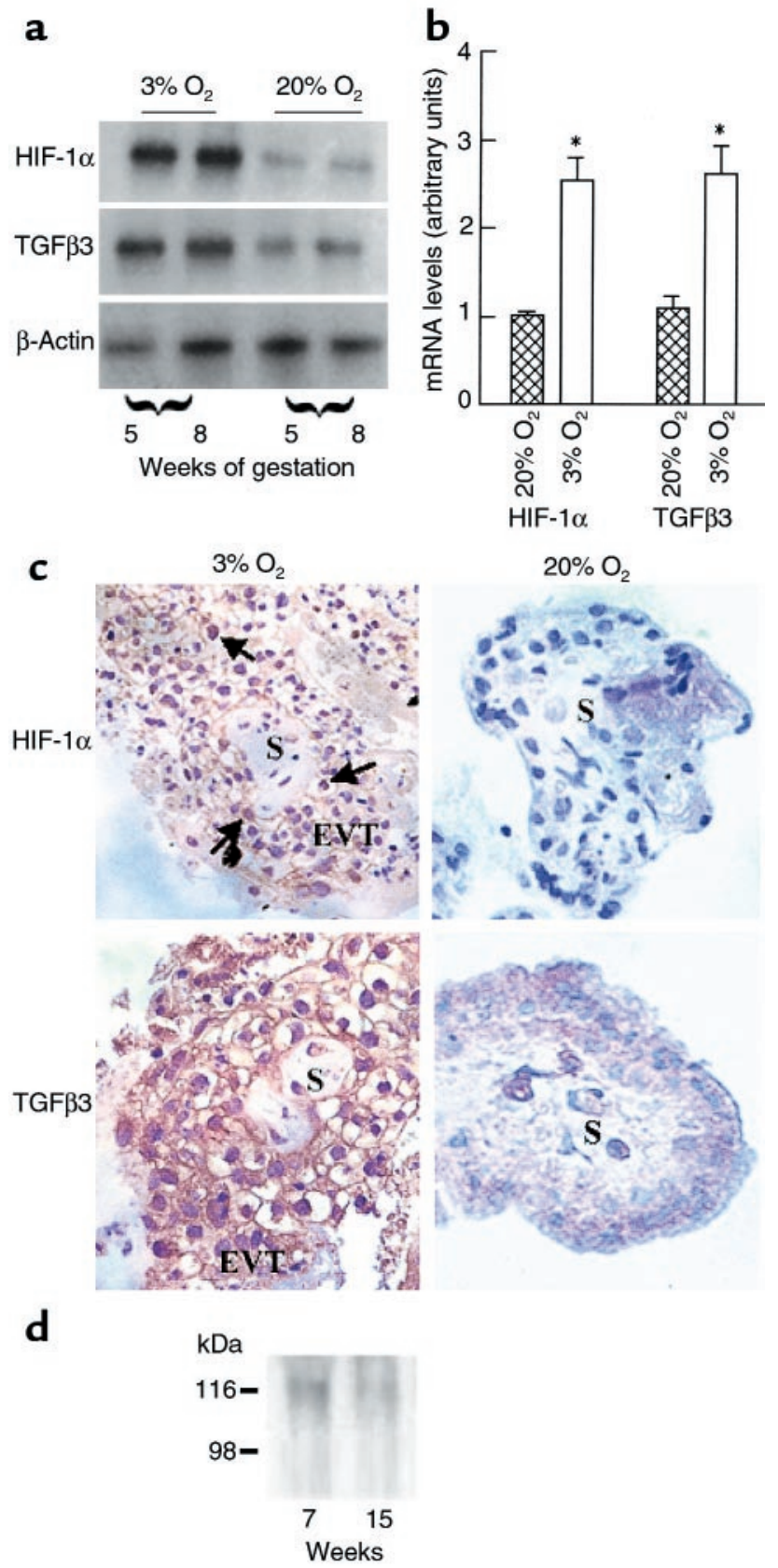

\section{Figure 3}

Expression of HIF- $1 \alpha$ villous explants exposed to different oxygen tension (a) Exposure of villous explants to 20\% $\mathrm{O}_{2}$ downregulates the mRNA expression for HIF- $1 \alpha$ and TGF $\beta_{3}$. (b) Bands were quantified by densitometric analysis. Shown are the changes in HIF- $1 \alpha$ and TGF $\beta_{3}$ mRNA after normalization to control cultures kept at $20 \% \mathrm{O}_{2}$. All data are expressed as the mean \pm SEM of 5 separate experiments carried out in triplicate. (c) Immunoperoxidase staining of HIF- $1 \alpha$ and TGF $\beta_{3}$ was performed in sections from explants kept at either $3 \%$ or $20 \% \mathrm{O}_{2}$. Sections of explants kept at $3 \% \mathrm{O}_{2}$ show positive HIF- $1 \alpha$ and $\mathrm{TGF} \beta_{3}$ immunoreactivity in EVT cells of the outgrowth (EVT), whereas no immunoreactivity for HIF- $1 \alpha$ and low immunoreactivity for TGF $\beta_{3}$ are noted in sections of explants kept at $20 \% \mathrm{O}_{2}$. S, villous stroma. (d) Western blot analysis for HIF- $1 \alpha$ in human placental tissue. HIF- $1 \alpha$ protein $(116 \mathrm{kDa})$ was detected in placentas of 7 weeks' gestation, but not 15 weeks' gestation. 

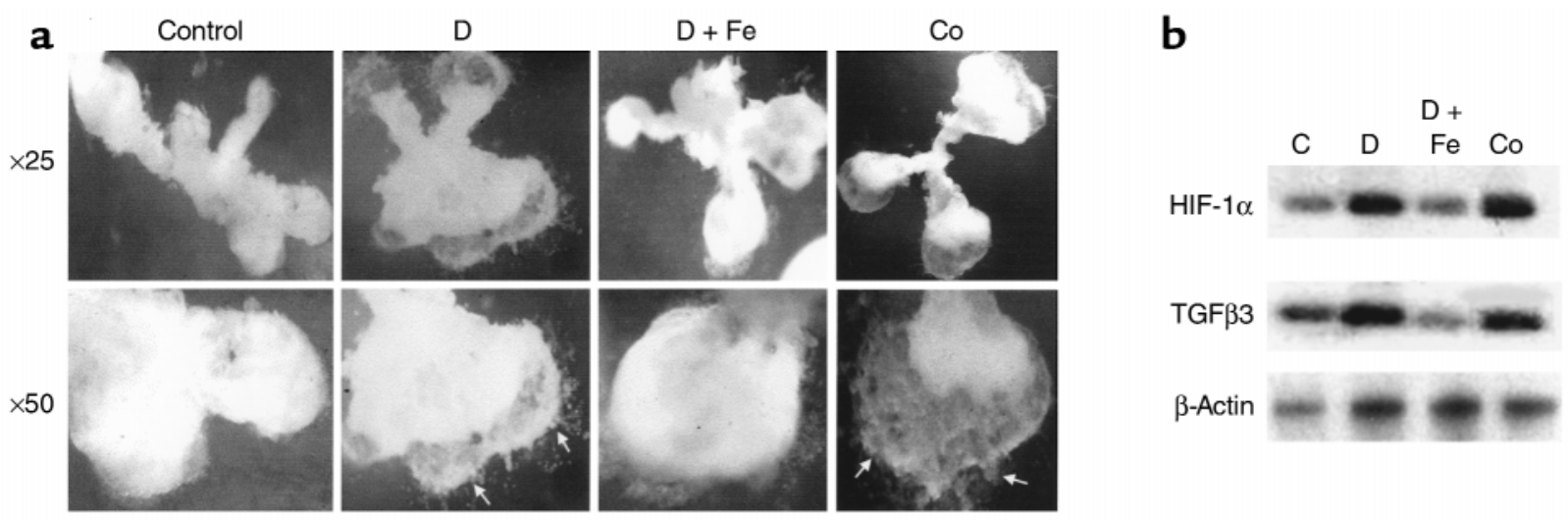

\section{Figure 4}

Desferoxamine and cobalt chloride mimic the low-oxygen effect on EVT outgrowth and HIF- $1 \alpha$ and TGF $\beta_{3}$ expression. (a) Exposure of villous explants, cultured in $20 \% \mathrm{O}_{2}$ to either desferoxamine $(10 \mu \mathrm{M})$ or $\mathrm{CoCl}_{2}(100 \mu \mathrm{M})$ increased EVT outgrowth (arrows). Addition of FeCl 2 $(20 \mu \mathrm{M})$ to the desferoxamine-treated cultures prevented the outgrowth. (b) Exposure of villous explants to either desferoxamine (10 $\mu \mathrm{M})$ or cobalt chloride $(100 \mu \mathrm{M})$ increased mRNA expression for HIf-1 $\alpha$ and TGF $\beta_{3}$. C, control medium; D, desferoxamine; Co, cobalt chloride; $\mathrm{D}+\mathrm{Fe}$, desferoxamine + iron chloride. $\times 25, \times 50$.

sion of HIF- $1 \alpha$ mRNA exhibited a striking pattern of developmental regulation with levels high at 5-8 weeks, but declining markedly at 9 weeks of gestation and absent at 11-14 weeks. HIF-1 $\alpha$ mRNA was predominantly localized to the trophoblast layers with limited expression in the mesenchyme (Figure 2a). Western blot analysis revealed that the HIF- $1 \alpha \mathrm{mAb}$ recognized a protein of approximately $116 \mathrm{kDa}$ (Figure $3 \mathrm{~d}$ ), in agreement with previous studies (18). HIF- $1 \alpha$ protein showed a similar developmental expression pattern as HIF- $1 \alpha$ mRNA, i.e., high at 7 weeks but low/absent at 15 weeks of gestation.

Effect of low oxygen tension on HIF- $1 \alpha$ and TGF $\beta_{3}$ expression in villous explants. To define whether HIF- $1 \alpha$ plays a role in modulating trophoblast function in low oxygen tension we studied the effect of different oxygen tensions on HIF- $1 \alpha$ gene expression in the villous explant system. Villous explants from 5 to 8 weeks' gestation, cultured at $3 \% \mathrm{O}_{2}$, demonstrated significantly greater mRNA expression of HIF- $1 \alpha$ when compared with explants cultured at $20 \% \mathrm{O}_{2}$ (Figure 3 , a and b). The effect of low $\mathrm{pO}_{2}$ on HIF-1 $\alpha$ gene expression was noted as early as at 24 hours of incubation (data not shown). Because the ontogeny of placental HIF- $1 \alpha$ mRNA expression (Figure 2) was similar to that of TGF $\beta_{3}(14)$, we also determined the effect of low oxygen tension on $\mathrm{TGF}_{3}$ mRNA expression in villous explants. Three percent oxygen upregulated the number of TGF $\beta_{3}$ transcripts when compared with $20 \% \mathrm{O}_{2}$ (Figure 3 , a and b). The abundant expression of HIF- $1 \alpha$ and TGF $\beta_{3}$ in villous explants, cultured at $3 \% \mathrm{O}_{2}$, was further confirmed by histochemical analysis. Immunoreactive HIF- $1 \alpha$ protein localized to the EVT in the low oxygen-induced outgrowth (Figure 3c). In contrast, explants cultured at $20 \% \mathrm{O}_{2}$ demonstrated no HIF- $1 \alpha$ immunoreactivity (Figure 3c). Positive immunoreactivity for TGF $\beta_{3}$ was observed in explants cultured at either 3 or $20 \% \mathrm{O}_{2}$; however, $\mathrm{TGF}_{3}$ immunoreactivity was markedly stronger in explants kept at 3\% $\mathrm{O}_{2}$ (Figure 3c).
To determine whether low $\mathrm{pO}_{2}$ regulates HIF- $1 \alpha$ and $\mathrm{TGF}_{3}$ gene expression by way of an iron (heme)-containing protein, villous explants were cultured at $20 \% \mathrm{O}_{2}$ in the presence of either cobalt chloride or the iron chelator desferoxamine, both of which interfere with binding of molecular oxygen to heme proteins, thus mimicking hypoxia. Explants cultured in the presence of either 100 $\mu \mathrm{M}$ cobalt chloride or $10 \mu \mathrm{M}$ desferoxamine showed an increase in the EVT outgrowth (Figure 4a) similar to that observed with explants maintained at $3 \% \mathrm{O}_{2}$ (Figure 1a). Treatment of villous explants with either cobalt chloride or desferoxamine resulted in an increase of both HIF- $1 \alpha$ and TGF $\beta_{3}$ mRNA expression (Figure $4 \mathrm{~b}$ ). Addition of iron $(20 \mu \mathrm{M})$ to the desferoxamine-treated explants reversed both the stimulatory effect on EVT outgrowth (Figure 4a) and HIF-1 $\alpha$ and TGF $\beta_{3}$ mRNA expression (Figure $4 \mathrm{~b}$ ). These data suggest that hypoxia upregulates HIF- $1 \alpha$ and TGF $\beta_{3}$ gene expression in human villous explants and that this is mediated by way of a ferroprotein-dependent mechanism.

Antisense inbibition of HIF-1 $\alpha$ downregulates TGF $\beta_{3} m R N A$ expression in villous explants. To determine whether HIF- $1 \alpha$ mediates the hypoxia-induced TGF $\beta_{3}$ expression, we examined HIF- $1 \alpha$ and TGF $\beta_{3}$ mRNA expression in explants exposed to antisense HIF- $1 \alpha$ and TGF $\beta_{3}$ oligonucleotides, respectively. Treatment of villous explants, cultured in $3 \% \mathrm{O}_{2}$ with antisense oligonucleotides to HIF$1 \alpha$ (but not sense oligonucleotide-treated or controluntreated explants) abolished both TGF $\beta_{3}$ and HIF- $1 \alpha$ mRNA expression (Figure $5, \mathrm{a}-\mathrm{c}$ ), but not that of $\beta$-actin (Figure 5, a and b). In contrast, treatment of villous explants with antisense oligonucleotides to $\mathrm{TGF}_{3}$ abolished TGF $\beta_{3}$ mRNA expression but not that of HIF- $1 \alpha$ (Figure $5 b$ ). These data corroborate the specificity of the antisense HIF-1 $\alpha$ effect and suggest that HIF-1 $\alpha$ may regulate $\mathrm{TGF} \beta_{3}$ expression during early EVT outgrowth.

Antisense inbibition of HIF-1 $\alpha$ expression triggers a switch from a proliferative to an invasive trophoblast phenotype. To determine the functional significance of HIF- $1 \alpha$ expres- 

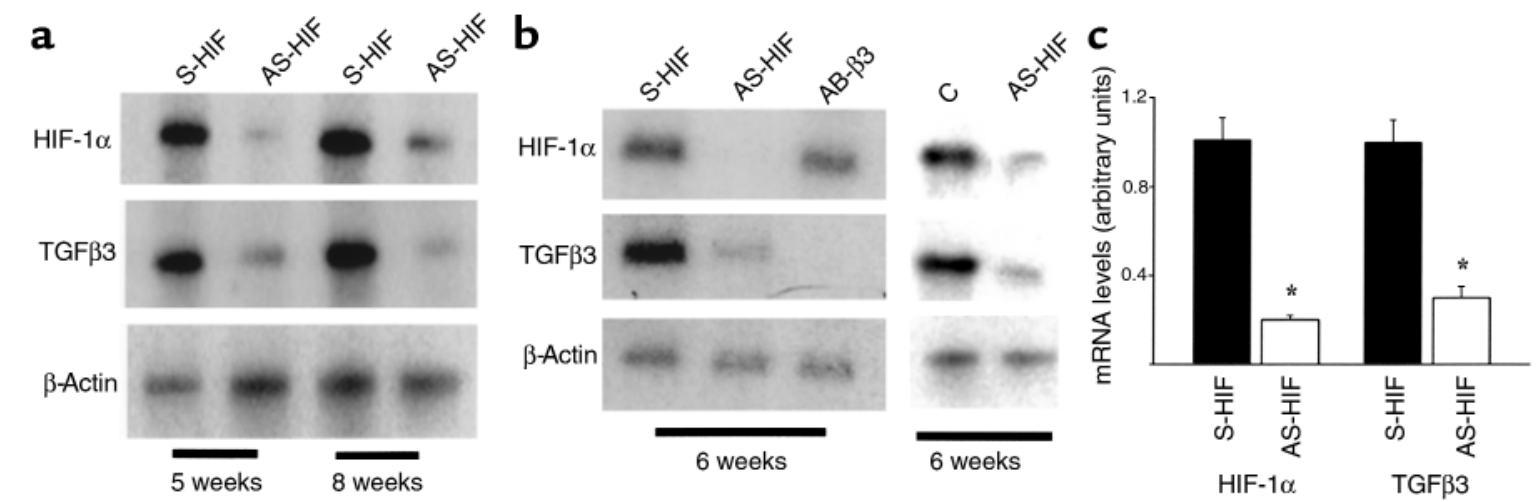

Figure 5

Antisense oligonucleotides to HIF- $1 \alpha$ inhibit HIF- $1 \alpha$ and TGF $\beta_{3}$ mRNA expression. Explants of 5-8 weeks' gestation were treated for 5 days with $10 \mu \mathrm{M}$ antisense oligonucleotides to HIF- $1 \alpha$ (AS-HIF) and antisense oligonucleotides to TGF $\beta_{3}$ (AS- $\beta 3$ ). Control experiments were run in parallel using either sense oligonucleotides to HIF- $1 \alpha$ (S-HIF) and TGF- $\beta_{3}$ (S- $\beta 3$ ) or medium alone (C). Expression of HIF-1 $\alpha$ and TGF $\beta_{3}$ mRNA was measured by low-cycle RT-PCR followed by Southern blot analysis using specific probes for HIF- $1 \alpha$, TGF- $\beta_{3}$, and the control housekeeping gene $\beta$-actin. (a) Antisense HIF- $1 \alpha$ treatment of villous explants inhibits both HIF- $1 \alpha$ and TGF $\beta_{3}$ mRNA. (b) Antisense TGF $\beta_{3}$ treatment of villous explants inhibits TGF $\beta_{3}$ mRNA expression but not that of HIF- $1 \alpha$, whereas antisense HIF- $1 \alpha$ exposure results in inhibition of both HIF- $1 \alpha$ and TGF $\beta_{3}$ mRNA expression. (c) Bands were quantified by densitometric analysis. Shown are the changes in HIF- $1 \alpha$ and $\mathrm{TGF} \beta_{3}$ mRNA after normalization to control cultures using sense oligonucleotides. All data are expressed as the mean \pm SEM of 3 separate experiments carried out in triplicate.

sion during early placentation, we monitored trophoblast response to antisense-induced inhibition of HIF-1 $\alpha$ expression in explants at $5-8$ weeks of gestation. EVT outgrowth and proliferation without cell invasion was visible in control explants kept in $3 \% \mathrm{O}_{2}$ in the presence of medium alone or sense oligonucleotides (Figure 6a). In contrast, explants exposed to antisense HIF- $1 \alpha$ oligonucleotides displayed prominent EVT outgrowth from the distal end of the villous tip and an increased number of columns of cells migrating and invading into the surrounding matrix (Figure 6a). We next performed experiments to characterize the invasive phenotype of trophoblast cells in the outgrowth of the antisense-treated explants to confirm that trophoblasts in the outgrowth underwent a differentiation pathway typical of the extravillous trophoblast cells in vivo. Villous explants kept at 3\% $\mathrm{O}_{2}$ showed an increase in newly synthesized fibronectin after 5 days of culture (Figures $1 \mathrm{a}$ and $6 \mathrm{~b}$ ). In contrast, a
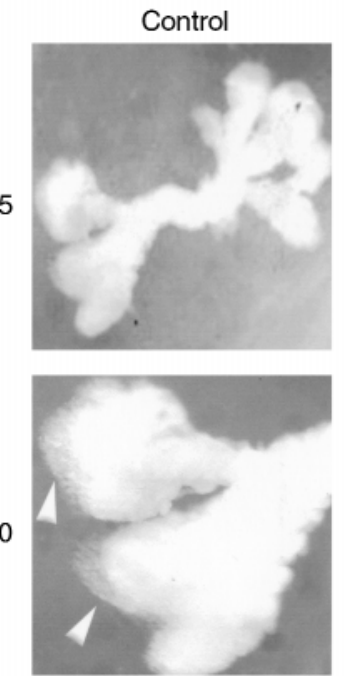

S-HIF
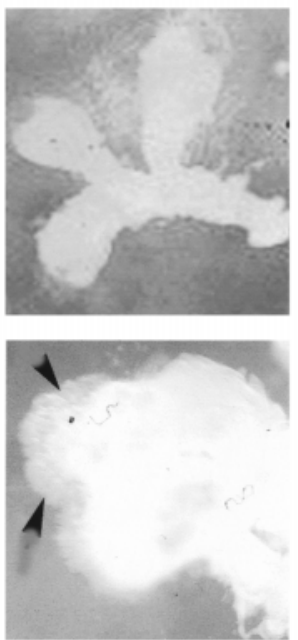

AS-HIF
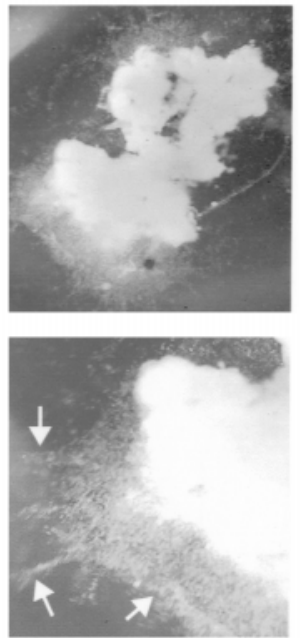

b
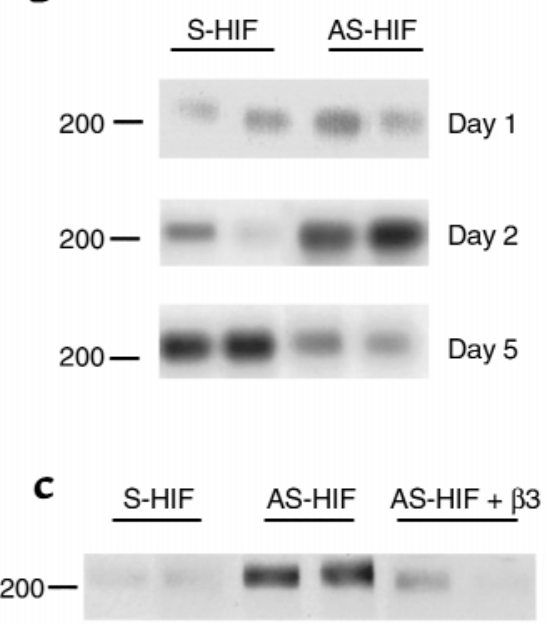

Figure 6

Antisense oligonucleotides to HIF- $1 \alpha$ induce extravillous trophoblast invasion and fibronectin production in villous explant cultures. (a) Villous explants of 5-8 weeks' gestation were maintained in culture for 5 days at $3 \% \mathrm{O}_{2}$ in the presence of $10 \mu \mathrm{M}$ antisense oligonucleotides to $\mathrm{HIF-1} \alpha$ (AS-HIF). Control experiments were run in parallel using explants from the same placentas cultured with medium alone or medium containing sense oligonucleotides (S-HIF). Note that antisense HIF-1 $\alpha$-treatment dramatically increases EVT invasion into the surrounding Matrigel (arrows) when compared with control villous explants, which show the typical low $\mathrm{pO}_{2}$-induced outgrowth (arrowheads). $\times 25$, $\times 50$. (b) Antisense HIF$1 \alpha$ treatment increases fibronectin synthesis as early as 2 days after oligonucleotide exposure. Note that after 5 days of treatment the antisensestimulatory effect on fibronectin synthesis is lost. (c) Shown is a representative experiment demonstrating that addition of recombinant TGF $\beta_{3}$ to the antisense HIF-1 $\alpha$-treated explants abolished the early antisense-stimulatory effect on fibronectin production. 

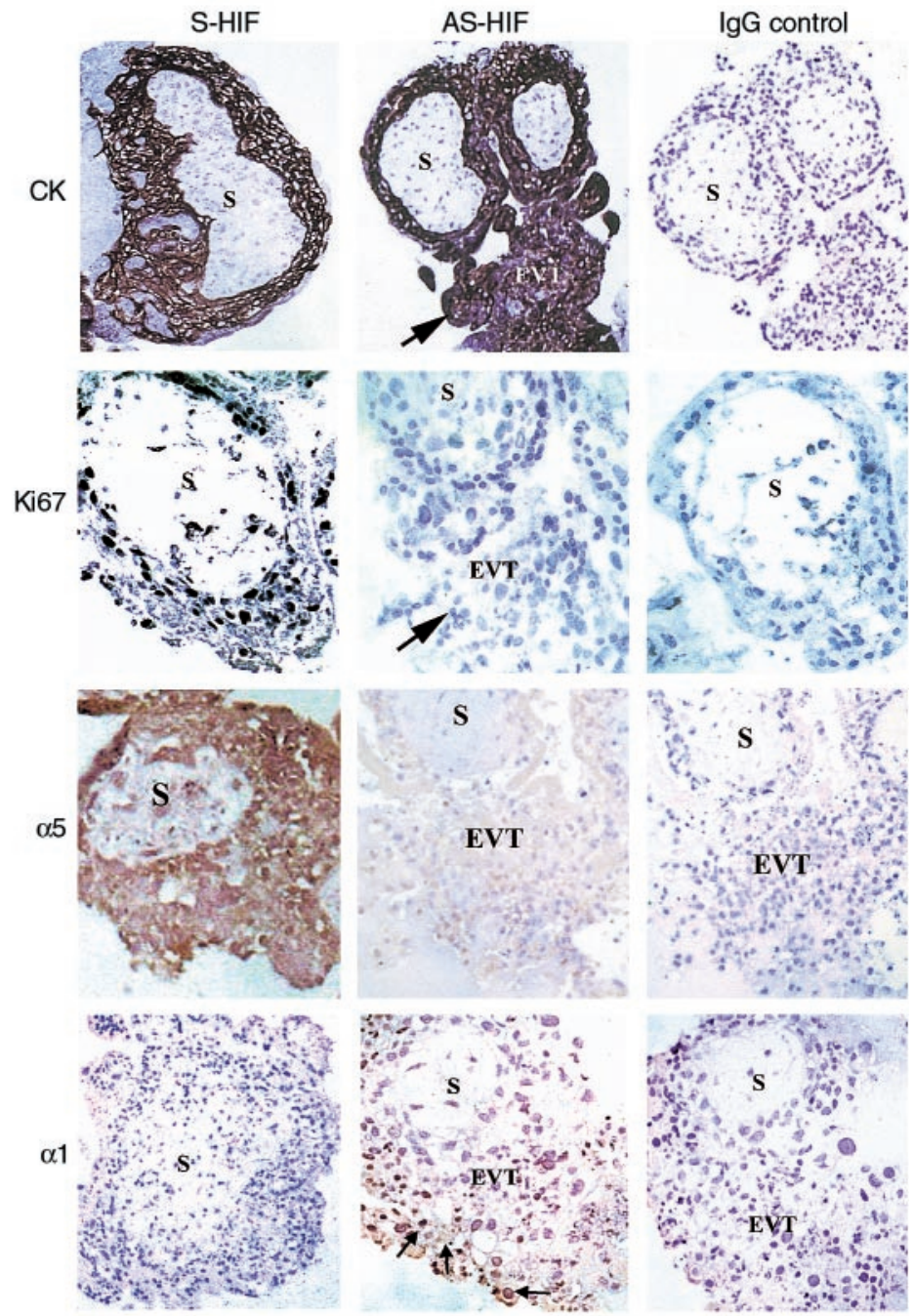

Figure 7

Antisense oligonucleotides to HIF- $1 \alpha$ inhibit proliferation and $\alpha_{5}$ integrin expression and induce $\alpha_{1}$ integrin expression in villous explant cultures. Immunoperoxidase staining of cytokeratin, Ki67, integrin $\alpha_{5}$, and $\alpha_{1}$ integrin was performed in placental sections from villous explants cultured under hypoxic condition with antisense and control sense oligonucleotides to HIF- $1 \alpha$. Sections of explants, cultured with antisense oligonucleotides, have no positive Ki67 immunoreactivity and markedly reduced staining for $\alpha_{5}$ integrin but are immunopositive for $\alpha_{1}$ integrin in EVT cells of the invading column (arrow, EVT). Sections of control cultures in the presence of sense oligonucleotides show positive immunoreactivity for Ki67 and $\alpha_{5}$ integrin but no positive $\alpha_{1}$ integrin staining in the EVT of the outgrowth induced by $3 \% \mathrm{O}_{2}$ exposure. Independent of treatment, trophoblast cells stained positive for cytokeratin (CK), a marker for epithelial cells. Control immunostaining experiments were performed using control lgG. S, villous stroma.

proliferative phenotype (Figure 7). All trophoblast cells stained positively for cytokeratin, indicating the epithelial-like nature of the proliferating trophoblast cells in the low oxygen tension-induced outgrowth as well as in the invasive column of EVT after antisense HIF- $1 \alpha$ treatment (Figure 7).

Antisense treatment markedly increased MMP9 immunoreactivity in the EVT within the outgrowth. Control sense oligonucleotide-treated explants showed weak immunoreactivity for MMP9 in the villous cytotrophoblast and in the stroma (Figure 8a). Gelatin zymography analysis of conditioned media from sense- and antisense-treated explants after 1 day of culture revealed gelati-

explants kept at $3 \% \mathrm{O}_{2}$ in the presence of antisense oligonucleotides to HIF- $1 \alpha$ showed increased fibronectin production as early as 2 days of culture with a decrease thereafter, suggesting that downregulation of HIF-1 $\alpha$ results in trophoblast differentiation along the invasive pathway (Figure 6b). Of interest, addition of recombinant $\mathrm{TGF}_{3}$ to the antisense HIF- $1 \alpha$-treated explants reversed the antisense stimulatory effect on fibronectin synthesis, which is in agreement with $\mathrm{TGF}_{3}$ being downstream of HIF-1 $\alpha$ (Figure $6 c$ ). Similar to previous reports $(5,22)$, exposure of first-trimester villous explants to low oxygen tension resulted in increased proliferation (assessed by Ki67 immunoreactivity) at the proximal site of the column before trophoblasts start to migrate away from the anchoring villus (Figures $1 \mathrm{~b}$ and 7 ). However, when explants in low oxygen tension were exposed to antisense HIF- $1 \alpha$ oligonucleotides, we observed stimulated EVT outgrowth together with an increase in expression of biochemical markers of the invasive trophoblast phenotype such as gelatinase $\mathrm{B} / \mathrm{MMP} 9(23,24)$ and $\alpha_{1}$ integrin $(24)$ (Figures 7 and 8), the latter being found in the EVT at the distal edge of the Matrigel (Figure 6). At the same time downregulation of HIF- $1 \alpha$ resulted in markedly reduced Ki67 and $\alpha 5$ integrin immunoreactivity, suggesting a less nase A/MMP2 activities at 60 and $68 \mathrm{kDa}$. After 3 days of culture, exposure of villous explants to antisense HIF$1 \alpha$ oligonucleotides resulted in an increase of gelatinase A/MMP2 activity and appearance of gelatinase B/MMP9 activities at 84 and $92 \mathrm{kDa}$ (Figure 8b). It is interesting that after 5 days of culture antisense-HIF- $1 \alpha$ oligonucleotide treatment decreased gelatinase A/MMP2 activity and increased gelatinaseB/MMP9 activity when compared with control cultures kept at $3 \%$ $\mathrm{O}_{2}$ in the presence of sense oligonucleotides (Figure 8b). Western blot analysis confirmed the upregulation of the MMP9 expression in explants treated for 5 days with antisense-HIF-1 $\alpha$ (Figure 8c).

\section{Discussion}

In this report we demonstrate that oxygen tension is a critical regulator of trophoblast differentiation and that HIF- $1 \alpha$ is upstream of TGF $\beta_{3}$ in mediating the effects of oxygen upon EVT differentiation. This conclusion is based on 3 observations: first, the parallel expression patterns of TGF $\beta_{3}$ and HIF- $1 \alpha$ are seen during EVT development; second, increasing oxygen tension downregulates expression of both HIF- $1 \alpha$ and $\mathrm{TGF} \beta_{3}$; and third, the trophoblast invasive phenotype 
can be prematurely triggered in first trimester placentas by downregulation of TGF $\beta_{3}$ by way of antisense inhibition of HIF- $1 \alpha$.

During the invasion process, trophoblast cells undergo striking and rapid changes in cellular functions that are temporally and spatially regulated along the invasive pathway. During the formation of the anchoring villi, proliferating cytotrophoblasts break through the syncytium and form columns of nonpolarized extravillous trophoblast cells. This initial proliferative process is associated with a switch in the repertoire of integrins and extracellular matrix remodeling. EVT subsequently differentiate to acquire an invasive phenotype (25), expressing markers such as gelatinase B/MMP9 and $\alpha 1$ integrin $(23,26,27)$ that permit the cells to invade and surround the maternal vessels in the decidua. Using a human villous explant system, we demonstrated that incubation of villous explants from early first-trimester placentas in their assumed physiologic $\mathrm{pO}_{2}\left(3 \% \mathrm{O}_{2}\right)$ environment is accompanied by changes in EVT outgrowth, cell proliferation, fibronectin synthesis, and gelatinase A/MMP2 activity $(15,21)$. Our data agree with previous observations that persistent low oxygen tension arrests trophoblast differentiation at this initial proliferative noninvasive stage, characterized by $\alpha 5$ integrin expression and low $\alpha 1$ integrin expression $(5,28)$. In contrast, in explants cultured at $20 \%$ oxygen, cytotrophoblasts of the proximal column do maintain a proliferative state beyond 24 hours (29). Several other studies document responses of trophoblasts to low oxygen tension, including increased production of the inflammatory cytokines (e.g., TNF $\alpha$, IL- $1 \alpha$, and IL-1 $\beta[30,31]$ ), VEGF (32), plasminogen activator inhibitor-1 (33), and inhibition of cytotrophoblast differentiation toward syncytium (34).
HIF-1 $\alpha$ has been shown to be a major regulator of cell function and differentiation in a number of in vivo animal models and cell systems (10-13). Our data suggest that HIF- $1 \alpha$ may also mediate the effects of oxygen on human trophoblast development. Thus, expression of HIF- $1 \alpha$ mRNA in placental trophoblast is high early on between 5 to 8 weeks and then falls precipitously around 10 to 12 weeks, precisely at the time when the intervillous space is perfused by maternal blood and $\mathrm{pO}_{2}$ levels are believed to increase (4). Moreover, HIF$1 \alpha$ mRNA and protein are high in trophoblast of villous explants cultured under physiologic low oxygen tension, and this expression is dramatically decreased when explants are exposed to higher oxygen levels. Janatpour et al (35), using isolated second-trimester trophoblasts, recently reported that HIF- $1 \alpha$ expression was elevated after 60 hours of culture in both 2 and $20 \%$ oxygen. These data are surprising because reports from a wide variety of mammalian systems (including our own study on first-trimester trophoblasts) indicate that HIF- $1 \alpha$ is upregulated by hypoxia. It is possible that this unusual response is unique to secondtrimester trophoblasts. Alternatively, because it has been reported that stress can induce expression of HIF$1 \alpha(36)$, it may be that the particular conditions under which these isolated trophoblasts were cultured may have imposed a stress resulting in elevated HIF- $1 \alpha$ expression irrespective of the levels of oxygen tension.

The decrease in HIF- $1 \alpha$ expression in vivo is associated with the period of maximal trophoblast invasion into the maternal decidua. Interestingly, antisense inhibition of HIF-1 $\alpha$ expression induced a premature switch to the invasive phenotype in human villous explants at 6-8 weeks of pregnancy, similar to that which normally

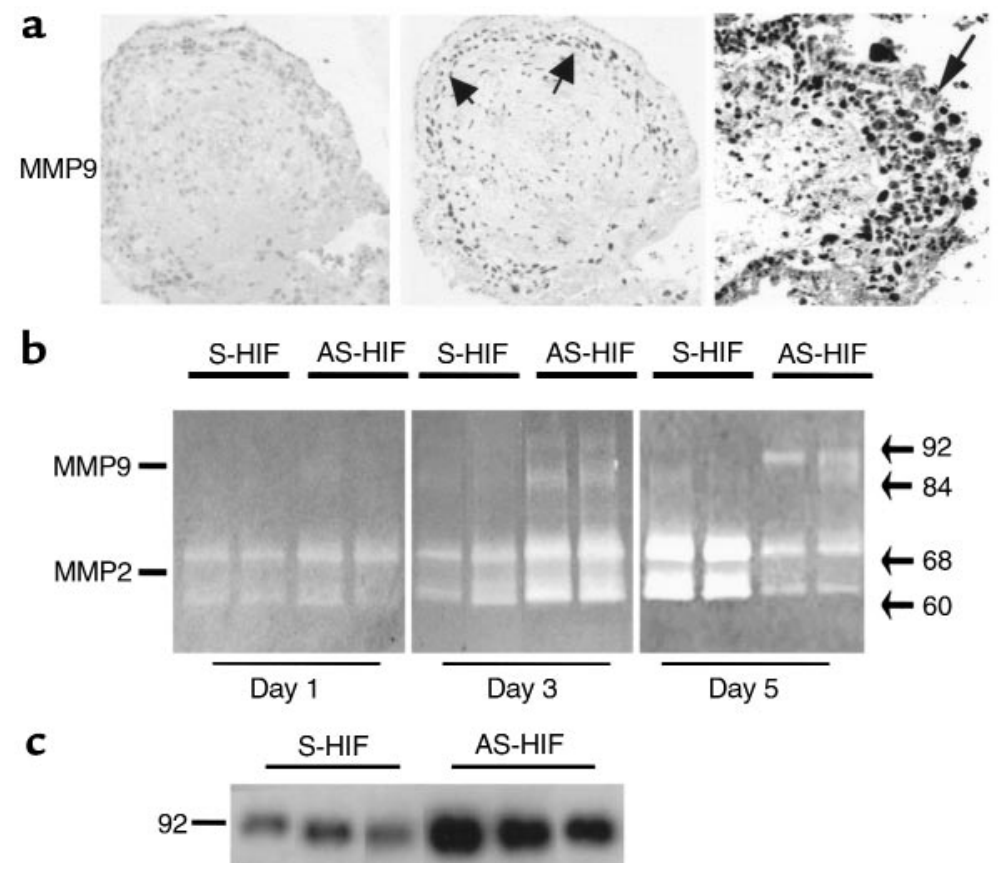

\footnotetext{
Figure 8

Antisense oligonucleotides to HIF- $1 \alpha$ trigger gelatinase expression and activity in villous explants cultured at $3 \% \mathrm{O}_{2}$. Explants of 5-8 weeks' gestation were treated with antisense (AS-HIF) or control sense (S-HIF) oligonucleotides to HIF-1 $\alpha$ (a) Section of explants treated for 5 days with antisense HIF- $1 \alpha$ show positive immunoreactivity for gelatinase B/MMP9 in the invading EVT (arrow, EVT) when compared with control sense-treated explants. Samples of conditioned medium were collected at days 1, 3, and 5 and subjected to analysis by gelatin zymography (b) or at day 5 for Western blotting with gelatinase B/MMP9 antisera (c). Arrows indicate positions of gelatinase activity (gelatinase A/MMP-2: 60 and $68 \mathrm{kDa}$; gelatinase B/MMP-9: 84 and $92 \mathrm{kDa}$ ).
} 
occurs at 10-12 weeks (when endogenous expression of HIF- $1 \alpha$ is reduced). This antisense HIF- $1 \alpha$-induced switch occurred despite the continued exposure of the explants to a low-oxygen environment that otherwise maintains them in a phase of arrested early development. We found that the switch is accompanied by inhibition of EVT proliferation, $\alpha_{5}$ integrin expression, a transient increase of fibronectin synthesis, MMP2 activity and an induction of MMP9, and $\alpha_{1}$ integrin expression. Note that treatment of explants with antisense HIF- $1 \alpha$ inhibited proliferation and early EVT events (5). This observation is consistent with a recent report showing that hypoxic exposure of isolated trophoblasts induces changes in the expression of proteins that control the cell cycle (37). Moreover, HIF-1 $\alpha$ has recently been reported to control proliferation and apoptosis of embryonic stem cells by regulating genes such as $p 53$, p21, and $\mathrm{Bcl}-2$ (36). Together these data suggest a key role for HIF- $1 \alpha$ in regulating trophoblast proliferation under conditions of low oxygen tension.

Data from gene-targeting studies also support a role for the HIF family of transcription factors in placental development and uterine vascular remodeling. The disruption of the HIF- $1 \alpha$ gene in mice results in embryonic lethality after cardiovascular and neuronal defects (38), whereas ARNT (HIF-1 $\beta$ )-deficient mice die after abnormalities related to angiogenesis (39) or from a failure in placental vascular development and formation of the labyrinthine spongiotrophoblast (40). The pattern of expression of HIF-1 $\alpha$ during the first trimester and the initiation of trophoblast differentiation in vitro upon knockdown of HIF- $1 \alpha$ levels paralleled data we published recently on $\mathrm{TGF}_{3}$ (14), leading us to investigate whether the effects of HIF- $1 \alpha$ were in fact mediated by TGF $\beta_{3}$. Our data support such a role. As with HIF$1 \alpha$, explants exposed to physiologic low oxygen tension expressed high levels of TGF $\beta_{3}$ mRNA and protein, but this expression was dramatically reduced upon exposure to high $(20 \%)$ oxygen. Moreover, in addition to triggering trophoblast differentiation along the invasive pathway, antisense knockdown of HIF- $1 \alpha$ expression led to inhibition of TGF $\beta_{3}$ expression. Critically, the effects of HIF-1 $\alpha$ knockdown could be reversed by $\mathrm{TGF} \beta_{3}$. The demonstration that antisense TGF $\beta_{3}$ treatment also induced markers of trophoblast invasion (14), but did not affect HIF-1 $\alpha$ expression, further supports this cytokine acting downstream of HIF- $1 \alpha$.

The mechanisms by which HIF- $1 \alpha$ expression is regulated in the trophoblast remain to be determined. A recent report showed that HIF- $1 \alpha$ protein is continuously synthesized but rapidly degraded in normoxia by the proteasome system by way of an irondependent pVHL/HIF-1 interaction $(13,41)$. Thus, it is possible that in addition to downregulation of HIF$1 \alpha$ mRNA expression degradation of HIF- $1 \alpha$ protein occurs in $20 \% \mathrm{O}_{2}$. Hypoxia, cobalt, iron chelators, and various antioxidants have been shown to stabilize the HIF- $1 \alpha$ subunit, thereby inducing the formation of the active HIF-1 complex $(13,41)$. However, the mech- anisms of oxygen sensing are yet unclear. Studies analysing the erythropoietin gene $(E P O)$, the first gene described to be responsive to hypoxia, have suggested that the oxygen sensor resides in a heme-containing molecule $(11,42)$. Herein, we demonstrate that treatment of villous explants, cultured at $20 \% \mathrm{O}_{2}$ with either cobalt chloride or the iron chelator desferoxamine, induced EVT outgrowth from the distal end of the villous tips and increased expression of HIF- $1 \alpha$ and $\mathrm{TGF}_{3}$ mRNA, thus mimicking the hypoxic response. Furthermore, we found that these effects were specific because addition of iron to the desferoxamine-treated explants abolished these stimulatory effects. Taken together, these data suggest that under reduced oxygen tension trophoblast cells activate HIF- $1 \alpha$, which in turn may upregulate $\mathrm{TGF}_{3}$ expression, and this oxygen effect appears to be mediated through a ferroprotein-dependent mechanism.

Mounting evidence suggests that the extravillous trophoblasts from early preeclamptic placentas are arrested at a relatively immature phenotype, possibly because of a failure to undergo complete differentiation along the invasive pathway during the first trimester of gestation. Preeclamptic placentas fail to complete integrin switching; i.e., the trophoblast remains positive for $\alpha_{5}$ and fails to express $\alpha_{1}$ (43), does not acquire an endovascular adhesion phenotype (44), demonstrates an excess of proliferative immature intermediate trophoblasts (45), and overexpresses fibronectin $(14,46)$. Data from our laboratory indicate that abnormalities in $\mathrm{TGF} \beta_{3}$ expression are associated with preeclampsia and that downregulation of $\mathrm{TGF} \beta_{3}$ with antisense oligonucleotides restores the invasive capability of preeclamptic trophoblasts (14).

In summary, our in vivo and in vitro HIF- $1 \alpha$ expression data strongly suggest that the early conceptus and placenta develop in a relative hypoxic environment. The data are further consistent with a model of normal placentation in which the placental $\mathrm{pO}_{2}$ increases around 10 to 12 weeks of gestation, reduces HIF- $1 \alpha$ expression, and in turn downregulates trophoblast TGF $\beta_{3}$ expression, thereby releasing the block to complete trophoblast differentiation into invasive EVT that invades deep into the maternal uterus. This invasion contributes to the remodeling of the uterine spiral arteries and, ultimately, enables the establishment of increased vascular perfusion of the placenta. A failure in a developmental switching in oxygen tension or a defect in the ability of the trophoblast to respond appropriately to this switch could result in abnormally elevated HIF- $1 \alpha$, and hence maintenance of TGF $\beta_{3}$ expression, for which we have shown results in an arrest of trophoblasts in a relatively immature state of differentiation. As a direct consequence, trophoblast invasion into the uterus is shallow, and uteroplacental perfusion is reduced. This may result in the clinical manifestations of preeclampsia, including shallow trophoblast invasion into the uterus and abnormally high uteroplacental vascular resistance. 


\section{Acknowledgments}

We thank Christine Botsford for providing the placental samples. We also thank John Kingdom, James Copeman, and Jay Cross for carefully reading the manuscript and Knox Ritchie for the constant support. This work was supported by the Department of Obstetrics and Gynaecology, Medical Research Council of Canada (MRC) grant MT-14096 (to I. Caniggia), and MRC Group Grant in Lung Development (to M. Post). I. Caniggia is an MRC Scholar.

1. Cross, J.C., Werb, Z., and Fisher, S.J. 1994. Implantation and the placenta: key pieces of the development puzzle. Science. 266:1508-1518.

2. Strickland, S., and Richards, W.G. 1992. Invasion of trophoblasts. Cell. 71:355-357.

3. Aplin, J.D. 1991. Implantation, trophoblast differentiation and haemochorial placentation: mechanistic evidence in vivo and in vitro. J. Cell Sci. 99:681-692.

4. Rodesch, F., Simon, P., Donner, C., and Jauniaux, E. 1992. Oxygen measurements in endometrial and trophoblastic tissues during early pregnancy. Obstet. Gynecol. 80:283-285.

5. Genbacev, O., Joslin, R., Damsky, C.H., Polliotti, B.M., and Fisher, S.J 1996. Hypoxia alters early gestation human cytotrophoblast differentiation/invasion in vitro and models the placental defects that occur in preeclampsia. J. Clin. Invest. 97:540-550.

6. Semenza, G.L., and Wang, G.L. 1992. A nuclear factor induced by hypoxia via de novo protein synthesis binds to the human erythropoietin gene enhancer at a site required for transcriptional activation. Mol. Cell. Biol. 12:5447-5454.

7. Forsythe, J.A., et al. 1996. Activation of vascular endothelial growth factor gene transcription by hypoxia inducible factor 1. Mol. Cell. Biol. 16:4604-4613

8. Semenza, G.L., Roth, P.H., Fang, H.-M., and Wang, G.L. 1994. Transcriptional regulation of genes encoding glycolytic enzymes by hypoxia inducible factor 1. J. Biol. Chem. 269:23757-23763.

9. Melillo, G., et al. 1995. A hypoxia responsive element mediates a novel pathway of activation of the inducible nitric oxide synthase promoter. $J$. Exp. Med. 182:1683-1693.

10. Wenger, R.H., and Gassmann, M. 1997. Oxygen(es) and the hypoxiainducible factor-1. J. Biol. Chem. 378:609-616.

11. Wang, G.L., and Semenza, G.L. 1993. General involvement of hypoxia inducible factor1 in transcriptional response to hypoxia. Proc. Natl. Acad. Sci. USA. 90:4304-4308.

12. Wang, G.L., Jiang, B.H., Rueand, E.A., and Semenza, G.L. 1995. Hypoxia inducible factor 1 is a basic helix-loop-helix PAS heterodimer regulated by cellular O2 tension. Proc. Natl. Acad. Sci. USA. 92:5510-5514.

13. Maxwell, P.H., et al. 1999. The tumor suppressor protein VHL targets hypoxia-inducible factors for oxygen-dependent proteolysis. Nature. 399:271-275.

14. Caniggia, I., Grisaru-Gravnoski, S., Kuliszewski, M., Post, M., and Lye, S.J. 1999. Inhibition of TGF $\beta_{3}$ restores the invasive capability of extravillous trophoblast in preeclamptic pregnancies. J. Clin. Invest. 103:1641-1650.

15. Caniggia, I., Taylor, C.V., Ritchie, J.W.K., Lye, S.J., and Letarte, M. 1997. Endoglin regulates trophoblast differentiation along the invasive pathway in human placental villous explants. Endocrinology. 138:4977-4988.

16. Malcolm, A.D. 1992. Uses of antisense nucleic acids: an introduction. Biochem. Soc. Trans. 20:745-746.

17. Wang, J., et al. 1995. Cloning and characterization of glucocorticoids induced genes in fetal rat lung fibroblasts: transforming growth factor B3. J. Biol. Chem. 270:2722-2728.

18. Camenisch, G., et al. 1999. General applicability of chicken egg yolk antibodies: the performance of $\operatorname{IgY}$ immunoglobulins raised against the hypoxia-inducible factor $1 \alpha$. FASEB J. 13:81-88.

19. Braissant, O., and Wahli, W. 1998. A simplified in situ hybridization protocol using non-radioactive labeled probes to detect abundant and rare mRNAs on tissue sections. Biochemica. 1:10-16.

20. Engvall, E., and Ruoslhati, E. 1977. Binding of soluble form of fibroblast surface protein, fibronectin, to collagen. Int. J. Cancer. 20:1-5.

21. Caniggia, I., Lye, S.J.L., and Cross, J.C. 1997. Activin is a local regulator of human cytotrophoblast cell differentiation. Endocrinology. 138:3976-3986

22. Zhou, Y., Genbacev, O., Damsky, C.H., and Fisher, S.J. 1998. Oxygen regulates human cytotrophoblast differentiation and invasion: implications for endovascular invasion in normal pregnancy and in pre-eclampsia. $J$. Reprod. Immunol. 39:197-213.

23. Fisher, S.J., et al. 1989. Adhesive and degradative properties of human placental cytotrophoblast cells in vitro. J. Cell Biol. 109:891-902.

24. Librach, C.L., et al. 1991. 92-kD type IV collagenase mediates invasion of human cytotrophoblast. J. Cell Biol. 113:437-449.

25. Zhou, Y., et al. 1997. Human cytotrophoblasts adopt a vascular phenotype as they differentiate. J. Clin. Invest. 99:2139-2151.

26. Damsky, C.H., et al. 1994. Integrin switching regulates normal trophoblast invasion. Development. 120:3657-3666.

27. Bischof, P., Redard, M., Gindre, P., Vassilakos, P., and Campana, A. 1993. Localization of alpha2, alpha5 and alpha6 integrin subunits in human endometrium, decidua and trophoblast. Eur. J. Obstet. Gynecol. Reprod. Biol. 51:217-226.

28. Fox, H., and Path, M.C. 1970. Effect of hypoxia on trophoblast in organ culture. Am. J. Obstet. Gynecol. 107:1058-1064.

29. Aplin, J.D., Haigh, T., Jones, C.J.P., Church, H.J., and Vicovac, L. 1999. Development of cytotrophoblast columns from explanted first-trimester human placental villi: role of fibronectin and integrin $\alpha 5 \beta 1$. Biol. Reprod. 60:828-838.

30. Benyo, D.F., Miles, T.M., and Conrad, K.P. 1997. Hypoxia stimulates cytokine production by villous explants from the human placenta. $J$. Clin. Endocrinol. Metab. 82:1582-1588.

31. Conrad, K.P., and Benyo, D.B. 1997. Placental cytokines and the pathogenesis of preeclampsia. Am. J. Reprod. Immunol. 37:240-249.

32. Taylor, C.M., Stevens, H., Anthony, F.W., and Wheeler, T. 1997. Influence of hypoxia on vascular endothelial growth factor and chorionic gonadotrophin production in trophoblast-derived cell lines: JEG, Jar and BeWo. Placenta. 18:451-458.

33. Fitzpatrick, T.E., and Graham, C.H. 1998. Stimulation of plasminogen activator inhibitor-1 expression in immortalized human trophoblast cells cultured under low levels of oxygen. Exp. Cell Res. 245:155-162.

34. Alsat, E., et al. 1996. Hypoxia impairs cell fusion and differentiation process in human cytotrophoblast, in vitro. J. Cell. Physiol. 168:346-353.

35. Janatpour, M.J., et al. 1999. A repertoire of differentially expressed transcription factors that offers insight into mechanisms of human cytotrophoblast differentiation. Dev. Genet. 25:146-157.

36. Carmeliet, P., et al. 1998. Role of HIF-1 $\alpha$ in hypoxia-mediated apoptosis, cell proliferation and tumour angiogenesis. Nature. 394:485-490.

37. Genbacev, O., Zhou, Y., Ludlow, J.W., and Fisher, S.J. 1997. Regulation of human placental development by oxygen tension. Science. 277:1669-1672.

38. Ryan, H.E., Lo, J., and Johnson, R.S. 1988. HIF-1 $\alpha$ is required for solid tumor formation and embryonic vascularization. EMBO J. 17:3005-3015.

39. Maltepe, E., Schmidt, J.V., Baunoch, D., Bradfieldand, C.A., and Simon, M.C. 1997. Abnormal angiogenesis and response to glucose and oxygen deprivation in mice lacking the protein ARNT. Nature. 386:403-407.

40. Kozak, K.R., Abbott, B., and Hankinson, O. 1997. ARNT deficient mice and placental differentiation. Dev. Biol. 191:297-305.

41. Salceda, S., and Caro, J. 1997. Hypoxia-inducible factor $1 \alpha$ (HIF-1 $\alpha)$ protein is rapidly degraded by the ubiquitin-proteasome system under normoxic conditions. Its stabilizatoin by hypoxia depends on redox-induced changes. J. Biol. Chem. 272:22642-22647.

42. Goldberg, M.A., Dunning, S.P., and Bunn, H.F. 1988. Regulation of the erythropoietin gene: evidence that the oxygen sensor is a heme protein. Science. 242:1412-1415.

43. Zhou, Y., Damsky, C.H., Chiu, K., Roberts, J.M., and Fisher, S.J. 1993. Preeclampsia is associated with abnormal expression of adhesion molecules by invasive cytotrophoblasts. J. Clin. Invest. 91:950-960.

44. Zhou, Y., Damsky, C.H., and Fisher, S.J. 1997. Preeclampsia is associated with failure of human cytotrophoblast to mimic a vascular adhesion phenotype. J. Clin. Invest. 99:2152-2164.

45. Redline, R.W., and Patterson, P. 1995. Pre-eclampsia is associated with an excess of proliferative immature intermediate trophoblast. Hum. Pathol. 26:594-600.

46. Kupferminc, M.J., Peaceman, A.M., Wigton, T.R., Rehnberg, K.A., and Socol, M.A. 1995. Fetal fibronectin levels are elevated in maternal plasma and amniotic fluid of patients with severe preeclampsia. Am. J. Obstet. Gynecol. 172:649-653. 